\title{
Concomitant Brugada-like and short QT electrocardiogram linked to SCN5A mutation
}

\author{
Kui Hong ${ }^{\star, 1,3}$, Jinzhu $\mathrm{Hu}^{1,3}$, Jianhua $\mathrm{Yu}^{1}$ and Ramon Brugada ${ }^{2}$
}

Mutations in the $\alpha$-subunit of cardiac sodium channel gene SCN5A can lead to the overlapping phenotypes of both the Brugada and type 3 long QT syndromes. However, the combination of Brugada and a short QT phenotype resulting from mutation in SCN5A has not previously been described. A man with concomitant Brugada-like and short QT electrocardiogram (ECG) was identified and the SCN5A gene was sequenced. Whole-cell patch clamp analysis of human embryo kidney (HEK) 293 cells expressing a SCN5A channel with the patient's sequence was used to investigate the biophysical properties of the channel. The patient with the family history of sudden death showed Brugada-like and short QT interval ECG. Sequence anlaysis of the coding region of the SCN5A gene, identified a G to A heterozygous missense mutation at nucleotide site 2066 that resulted in a amino-acid substitution of arginine to histidine at amino-acid site 689 (R689H). Patch clamp analysis showed that the R689H failed to generate current when heterologously expressed in HEK293 cells, indicating it was a loss-of-function mutation. Our finding firstly showes that a heterozygous missense mutation R689H in SCN5A gene results in the loss of protein function and the coexistents of the Brugada-like and short QT interval ECG phenotypes.

European Journal of Human Genetics (2012) 20, 1189-1192; doi:10.1038/ejhg.2012.63; published online 11 April 2012

Keywords: short QT; Brugada; mutation; SCN5A

\section{INTRODUCTION}

Brugada syndrome (BrS) is an electrical disorder of the heart that does not result from abnormal heart structure and is characterized by the coved ST segment elevation in the right precordial leads. Short QT syndrome (SQTS) clinically first described in 2000 has been associated with mutations in three potassium channel genes $(\mathrm{KCNH} 2$, $K C N Q 1$, and KCNJ2) that increase the activity (gain-of-function) of the channels. ${ }^{1-3}$ In 2007, loss-of-function mutations in the CACNA1C (A39V and G490R) and CACNB2 (S481L) genes encoding the alphal- and beta2b-subunits of the L-type calcium channel were reported in patients with short QT interval and Brugada-like electrocardiogram (ECG). ${ }^{4}$

The QT interval is determined by the cardiac action potential, which is balanced between inward and outward currents of myocardial cells. Genetic testing has shown that mutations in cardiac ion channels are responsible for an ever-increasing number and diversity of familial cardiac arrhythmia syndromes. For example, mutations in the $\alpha$-subunit of sodium channel gene (SCN5A) have been found in BrS associated with type 3 long QT syndrome (LQTS), ${ }^{5}$ sick sinus syndrome, ${ }^{6}$ atrial standstill, ${ }^{7}$ cardiac conduction disturbance, ${ }^{8}$ and atrial fibrillation. ${ }^{9}$

In this study, we describe the overlapping phenotypes of short QT interval with Brugada-like ECG in a Chinese man. Furthermore, we identify a heterozygous missense mutation in the patient's SCN5A sodium channel and show that this mutation (R689H) is a loss-of-function mutation possibly causing the cardiac defects.

\section{MATERIALS AND METHODS}

The study was carried out in accordance with the Code of Ethics of the World Medical Association (Declaration of Helsinki) and approved by the hospital review board. The patient gave written informed consent.

\section{Clinical Evaluation}

The patient underwent detailed clinical examinations including baseline of 12-lead ECG, echocardiography and laboratory test. The patient did not permit electrophysiological studies to be performed.

\section{Molecular genetics}

Genomic DNA was extracted from peripheral lymphocytes. All exon coding regions of disease-causing genes of SQTS (KCNQ1, KCNH2, KCNJ2, CACNA1C, CACNB2) and SCN5A were amplified by polymerase chain reaction (PCR) using primers that would amplify intronic flanking sequences. The PCR products were directly sequenced using a Bigdye Terminator Mix (Applied Biosystems, Carlsbad, CA, USA) in both strands and analyzed by cycle sequencing using an automated DNA sequencer (ABI Prism 3130XL; Applied Biosystems).

\section{Mutagenesis}

The mutation in SCN5A that altered an arginine to a histidine (R689H) was generated by site-directed mutagenesis of the plasmid pcDNA-SCN5A, which contained SCN5A cDNA cloned into pcDNA3.1 + (Invitrogen, Carlsbad, CA, USA). The following mutagenic sense and anti-sense primers were used to generate the R689H mutation: sense primer: $5^{\prime}$-TGCTGGAACCATCTCGCCC AGCGCTACCTG-3'; anti-sense primer: $5^{\prime}$-CTGGGCGAGATGGTTCCAGC ATGGTGGAC- $3^{\prime}$. The mutated plasmid was sequenced to ensure the presence

${ }^{1}$ Cardiology Department, The Second Affiliated Hospital of Nanchang University, Nanchang, China; ${ }^{2}$ Cardiovascular Genetics Center, UdG-IdIBGi, University of Girona, Girona, Spain

${ }^{*}$ Correspondence: Dr K Hong, Cardiology Department, The Second Affiliated Hospital of Nanchang University, Nanchang of JiangXi, Nanchang, 330006, China; Tel: + 86791 6312927; Fax: + 86791 6262262; E-mail: hongkui88@163.com

${ }^{3}$ These authors contributed equally to this work.

Received 7 November 2011; revised 18 January 2012; accepted 9 March 2012; published online 11 April 2012 
of the R689H mutation as well as the absence of other substitutions introduced by PCR.

\section{Patch clamp}

The R689H mutation was expressed in the human embryo kidney (HEK) 293 cells and characterized by whole-cell patch clamp recordings as previously described. ${ }^{1}$ Membrane currents were measured with the EPC 10 amplifier (HEKA Instruments Inc., Bellmore, NY, USA).The pipette solution contained (mmol/l): CsF 110, CsCl 20, NaF 10, EGTA 10, and HEPES 10 (pH 7.35 adjusted with $\mathrm{CsOH}$ ). The bath solution consisted of (mmol/l): $\mathrm{NaCl} 145$, $\mathrm{KCl} 4, \mathrm{MgCl}_{2} 1.0, \mathrm{CaCl}_{2} 1.8$, glucose 10, and HEPES 10 (pH 7.35 adjusted with $\mathrm{NaOH})$. Recordings were made at room temperature.

Voltage-dependent sodium currents were recorded at various membrane potentials from $-80 \mathrm{mV}$ to $60 \mathrm{mV}$ in $10 \mathrm{mV}$ increment for $50 \mathrm{~ms}$, holding potential was $-120 \mathrm{mV}$. Steady-state activation was estimated by measuring the peak sodium currents. Conductance $\mathrm{G}(\mathrm{v})$ was calculated by the equation: $\mathrm{G}(\mathrm{v})=\mathrm{I} /(\mathrm{Vm}-\mathrm{Erev}$ ) (I: peak currents, Vm: membrane potentials), Eres: the measured reversal potentials). Steady-state inactivation was estimated by a prepulse protocol that was a various membrane potentials from $-140 \mathrm{mV}$ to $-50 \mathrm{mV}$ for $500 \mathrm{~ms}$ followed by depolarizing to $-20 \mathrm{mV}$ for $20 \mathrm{~ms}$. Data for steady-state activation and steady-state inactivation were fitted with the Boltzmann equation.

\section{Data Acquisition and Analysis}

All signals were acquired at $20 \mathrm{kHz}$ with Patchmaster software (HEKA Instruments Inc., Bellmore, NY, USA). The data were analyzed with Fitmaster, Sigmaplot 10.0 and EXCEL data handling. Results were presented as mean \pm SE. Statistical analysis was performed with $t$-test and $P<0.05$ was considered statistically significant.

\section{RESULTS}

\section{Clinic and genetic data}

A 40-year-old man was admitted to our hospital with a ruptured quadriceps femoris tendon of the right knee. Cardiac analysis (12-lead ECG) indicated the patient had Brugada-like ECG (Figure 1) and was diagnosed by a cardiologist as also having a short QT interval $(\mathrm{QT}=320 \mathrm{~ms}, \mathrm{HR}=71 \mathrm{bpm})$. Echocardiography and laboratory analysis including serum electrolytes were normal. There was a history of sudden death in the man's family (his father encountered sudden death at the age of 39 years from unknown cause) consistent with the Brugada-like and short QT interval ECG phenotypes resulting from a genetic mutation. All medicines were withdrawn.
To explore the possibility that the cardiac phenotypes may represent a mutation in the $S C N 5 A$ gene, we sequenced the coding regions of $S C N 5 A$ and identified a $\mathrm{G}$ to $\mathrm{A}$ substitution at nucleotide site 2066 that resulted in heterozygous missense mutation $\mathrm{R} 689 \mathrm{H}$ (amino-acid change of arginine to histidine, Figure 2a). The same mutation was not detected in the 200 control chromosomes of the same ethnic background (Figure $2 \mathrm{~b}$ ). There was no mutation found in KCNQ1, KCNH2, KCNJ2, CACNA1C, CACNB2 genes. More indepth analysis was not possible, as the patient refused to participate in a cardiac electrophysiological study and family members would not allow their DNA to be analyzed.

\section{Biophysical properties}

Patch clamp analysis was used to further explore how the SCN5A R689H mutation resulted in Brugada-like and short QT interval ECG. HEK293 cells were transfected with wild-type (WT) and mutant (R689H) SCN5A cDNA. Figure 3 showed the current-voltage relationships in WT and $\mathrm{R} 689 \mathrm{H}$ mutant sodium channel. There was no sodium current with the R689H mutant sodium channel indicating loss-of-function of the mutation.

\section{DISCUSSION}

The patient in this study had both Brugada-like and short QT interval ECG. However, to date he had no symptoms of heart disease, although there was a family history of sudden death of unknown origin. The lack of cardiac symptoms may result from the phenotypes of BrS and SQTS being only partially penetrant. These phenotypes often become more apparent in the presence of external factors, such as medication (sodium channel blocker), fever, ${ }^{10}$ or electrolyte disorder. Because the ECG of this patient was not the typical type 1 Brugada ECG pattern, it would of course have been very interesting to see the response to a sodium channel blocker. Unfortunately, the patient refused to do this test. There was no mutation in the KCNQ1, $K C N H 2, K C N J 2, C A C N A 1 C$ and CACNB2 genes that had been confirmed to cause SQTS. In this patient, the Brugada-like and short QT phenotypes appears to result from a single heterozygous missense mutation in the SCN5A protein.

It is clear that $S C N 5 A$ protein carries a large inward depolarizing current $\left(\mathrm{I}_{\mathrm{Na}}\right)$ during phase 0 of the cardiac action potential. The importance of $\mathrm{I}_{\mathrm{Na}}$ for the cardiac electrical activity is reflected by the high incidence of arrhythmias in cardiac sodium

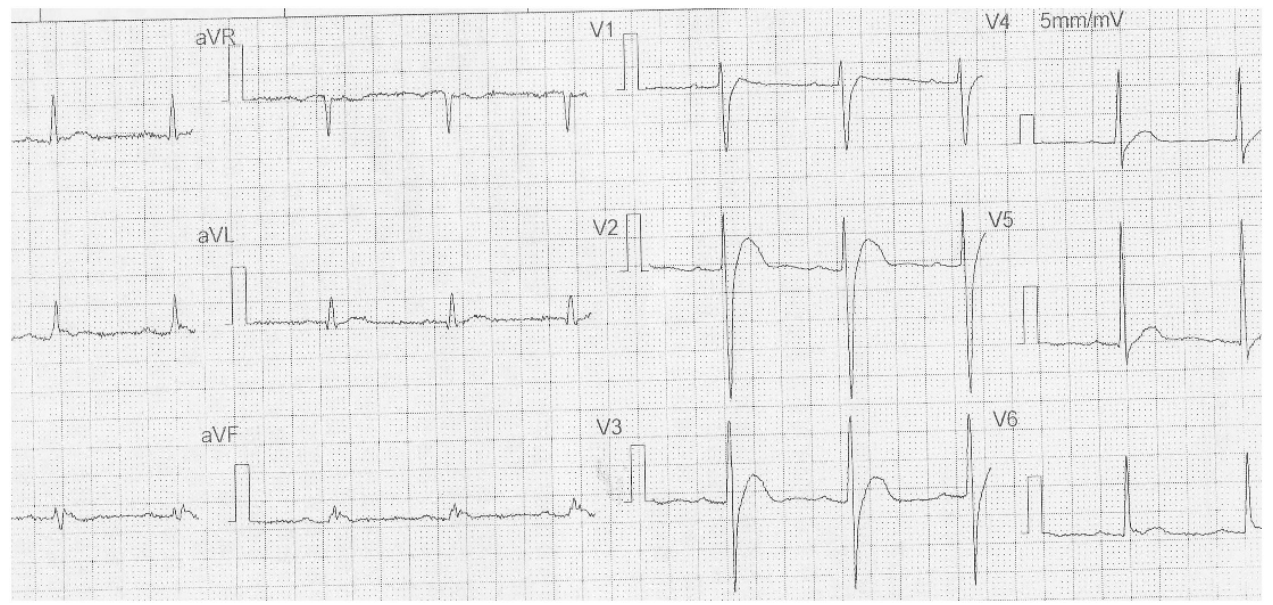

Figure 1 12-lead ECG $(5 \mathrm{~mm} / \mathrm{mV}, 25 \mathrm{~mm} / \mathrm{s})$ showed Brugada-like ST-T changes and short QT interval $(\mathrm{QT}=320 \mathrm{~ms}, \mathrm{HR}=71 \mathrm{bpm})$. 

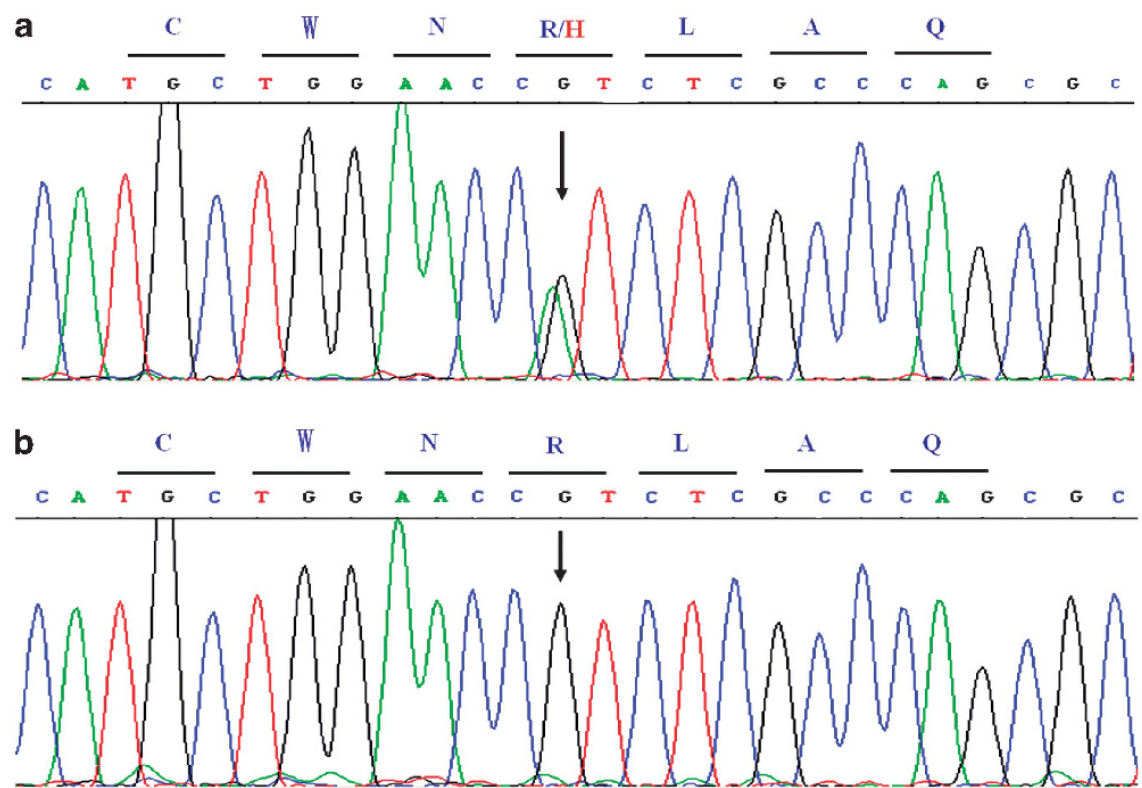

Figure 2 DNA sequence analysis of the patient with the SCN5A R689H heterozygous mutation (a) and a WT normal SCN5A sequence (b). The capital letters on the lines stand for amino-acids abbreviations.

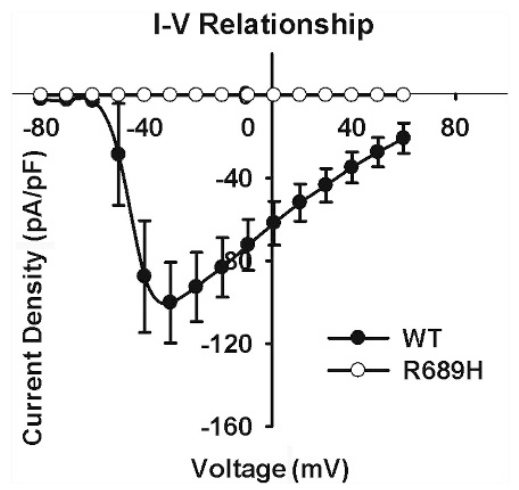

Figure 3 Current-voltage relationships for WT and R689H mutant sodium channel expressed in HEK293 cells. The peak value of I-V curve for WT occurred at $-30 \mathrm{mV}$, and the current density for $\mathrm{R} 689 \mathrm{H}$ was zero.

channelopathies. It is now recognized that reduced $\mathrm{I}_{\mathrm{Na}}$ decreases cardiac excitability and electrical conduction velocity, and induces BrS, progressive cardiac conduction disturbance, sick sinus syndrome, dilated cardiomyopathy, atrial fibrillation, sudden infant death syndrome, or combinations thereof. ${ }^{11}$ In our study, the $\mathrm{R} 689 \mathrm{H}$ mutation induced $\mathrm{I}_{\mathrm{Na}}$ dysfunction (zero current) that might result in the reduction of cardiac excitability and electrical conduction velocity, and therefore induce Brugada-like type ECG. It could also cause the abbreviation of the ventricular action potential resulting in a short QT interval. This finding is similar to another study in which blocking of the sodium channel shortened the QT but exacerbated the ST segment elevation of BrS phenotype causing enhanced risk for arrhythmia. ${ }^{12}$

The $\mathrm{R} 689 \mathrm{H}$ mutation has been described previously in patients with isolated LQTS $^{13}$ or BrS. ${ }^{15,16}$ Napolitano $\mathrm{C}^{13}$ found $\mathrm{R} 689 \mathrm{H}$ mutation in isolated LQTS probands (97\% White). Kapplinger JD ${ }^{15}$ and Nakajima $\mathrm{T}^{16}$ found $\mathrm{R} 689 \mathrm{H}$ mutation in isolated $\mathrm{BrS}$ patients (Hispanic and Japanese), respectively. The functional analysis of R689H mutation was not done in those three study. Although we found $\mathrm{R} 689 \mathrm{H}$ mutation associated with short QT interval and
Brugada-like ECG in a Chinese patient with the family history of sudden death and the functional analysis in HEK293 cell system did show the loss of function (no current). It is known that some genetic defects can lead to the overlapping syndromes in a single patient. ${ }^{14}$ This is the first report that a mutation in SCN5A causes a subclinical phenotype - Brugada-like and short QT interval ECG. The same mutation causing different manifestations suggested the complexity of genotype-phenotype relationship. The mechanism of this phenomenon was not well understood to date and it is commonly held that environmental and genetic modifiers presumably have a role in phenotype. ${ }^{17}$ The $\mathrm{R} 689 \mathrm{H}$ mutation caused LQTS in White race and caused the other different phenotypes in other race,which suggested that the ethnic genetic background have an important role in the arrhythmogenic phenotype susceptibility. ${ }^{18}$ The results of R689H mutation in HEK293 cells expression system only reflected the changes in HEK293 cells, which could not faithfully represent the function changes of $\mathrm{R} 689 \mathrm{H}$ heterozygous mutation. So the heterologous cell system had limited value in explaining the pathophysiological mechanism of the final comprehensive manifestation from a global patient. More sophisticated cell systems such as human stem cell-derived cell lines may be helpful to resolve these issues.

In conclusion, our finding is the first to show that a heterozygous missense mutation $\mathrm{R} 689 \mathrm{H}$ in SCN5A gene results in the loss of protein function and the coexistents of the Brugada-like and short QT interval ECG phenotypes.

\section{CONFLICT OF INTEREST}

The authors declare no conflict of interest.

\section{ACKNOWLEDGEMENTS}

We would like to thank Xiuxia Liu and Xin Yu for DNA extraction. This work was supported in part by the National Basic Research Program of China (973 Program: 2007CB512002 and 2008CB517305), the National Natural Science Foundation of China (30760076 and 81070148), and the JiangXi Scientific Foundation (2007BS02346). 
1 Brugada R, Hong K, Dumaine $\mathrm{R}$ et al: Sudden death associated with short-QT syndrome linked to mutations in HERG. Circulation 2004; 109: 30-35.

2 Bellocq C, van Ginneken AC, Bezzina CR et al: Mutation in the KCNQ1 gene leading to the short QT-interval syndrome. Circulation 2004; 109: 2394-2397.

3 Priori SG, Pandit SV, Rivolta I et al: A novel form of short QT syndrome (SQT3) is caused by a mutation in the KCNJ2 gene. Circ Res 2005; 96: 800-807.

4 Antzelevitch C, Pollevick GD, Cordeiro JM et al: Loss-of-function mutations in the cardiac calcium channel underlie a new clinical entity characterized by ST-segment elevation, short QT intervals, and sudden cardiac death. Circulation 2007; 115: 442-449.

5 Makita N, Behr E, Shimizu W et al: The E1784K mutation in SCN5A is associated with mixed clinical phenotype of type 3 long QT syndrome. J Clin Invest 2008; 118: 2219-2229.

6 Makiyama T, Akao M, Tsuji $\mathrm{K}$ et al: High risk for bradyarrhythmic complications in patients with Brugada syndrome caused by SCN5A gene mutations. J Am Coll Cardio 2005; 46: 2100-2106.

7 Takehara N, Makita N, Kawabe J et al: A cardiac sodium channel mutation identified in Brugada syndrome associated with atrial standstill. J Intern Med 2004; 255: 137-142.

8 Cordeiro JM, Barajas-Martinez $\mathrm{H}$, Hong $\mathrm{K}$ et al: Compound heterozygous mutations P336L and I1660V in the human cardiac sodium channel associated with Brugada syndrome. Circulation 2006; 114: 2026-2033.

9 Kusano KF, Taniyama M, Nakamura K et al: Atrial fibrillation in patients with Brugada syndrome relationships of gene mutation, electrophysiology, and clinical backgrounds. J Am Coll Cardiol 2008; 51: 1169-1175.
10 Patruno N, Pontillo D, Achilli A, Ruggeri G, Critelli G: Electrocardiographic pattern of Brugada syndrome disclosed by a febrile illness: clinical and therapeutic implications. Europace 2003; 5: 251-255.

11 Amin AS, Asghari-Roodsari A, Tan HL: Cardiac sodium channelopathies. Pflugers Arch 2010; 460: 223-237.

12 Priori SG, Napolitano C, Schwartz PJ, Bloise R, Crotti L, Ronchetti E: The elusive link between LQT3 and Brugada syndrome: the role of flecainide challenge. Circulation 2000; 102: 945-947

13 Napolitano C, Priori SG, Schwartz PJ et al: Genetic testing in the long QT syndrome:development and validation of an efficient approach to genotyping in clinical practice. JAMA 2005; 294: 2975-2980.

14 Makita N: Phenotypic overlap of cardiac sodium channelopathies: individual-specific or mutation-specific? Circ J 2009; 73: 810-817.

15 Kapplinger JD, Tester DJ, Alders M et al: An international compendium of mutations in the SCN5A-encoded cardiac sodium channel in patients referred for Brugada syndrome genetic testing. Heart Rhythm 2010; 7: 33-46.

16 Nakajima T, Kaneko Y, Saito A et al: Identification of six novel SCN5A mutations in Japanese patients with Brugada syndrome. Int Heart J 2011; 52: 27-31.

17 Scicluna BP, Wilde AA, Bezzina CR: The primary arrhythmia syndromes: same mutation, different manifestations. Are we starting to understand why? J Cardiovasc Electrophysiol 2008; 19: 445-452

18 Ackerman MJ, Splawski I, Makielski JC et al: Spectrum and prevalence of cardiac sodium channel variants among black, white, Asian, and Hispanic individuals: implications for arrhythmogenic susceptibility and Brugada/long QT syndrome genetic testing. Heart Rhythm 2004; 1: 600-607. 